\title{
Relating drug response to epigenetic and genetic markers using a region-based kernel score test
}

\author{
Summaira Yasmeen ${ }^{1 *}$, Patricia Burger ${ }^{1}$, Stefanie Friedrichs ${ }^{1}$, Sergi Papiol ${ }^{2,3}$ and Heike Bickeböller ${ }^{1}$
}

From Genetic Analysis Workshop 20

San Diego, CA, USA. 4 - 8 March 2017

\begin{abstract}
In GAW20, we investigated the association of specific genetic regions of interest (ROIs) with log-transformed triglyceride $(\mathrm{TG})$ levels following lipid-lowering medication using epigenetic and genetic markers. The goal was to incorporate kernels for cytosine-phosphate-guanine $(\mathrm{CpG})$ markers and compare the kernels to a purely parametric model. Post-treatment TG levels were investigated for post-methylation data at CpG sites and region-specific SNPs and adjusted for pre-treatment TG levels and age, in independent individuals only (real data: $n=150$; simulated data, replicate 84: $n=111$ ). In both data sets, our single-CpG-marker results using kernels and linear regression were in good agreement. In the real data, we investigated the introns of the CPT1A gene previously reported as associated with TG levels as separate ROls, and were able to find hints of an association of cg17058475 and cg00574958 with post-treatment TG levels. In the simulated data, we investigated a total of 10 regions, in which the 5 causal and 5 non-causal markers lie, respectively, with increased methylation variances, yielding plausible results for the 3 window sizes. Overall, this indicates that kernels for $\mathrm{CpG}$ markers are feasible. An interaction regression model for the causal SNP with the nearest CpG marker identified an effect for the SNPs with the three greatest heritabilities simulated. The simulation model assumed full SNP effect only for unmethylated regions decreasing to zero in the case of full methylation. Thus, in the context of a clear candidate setting, interaction between epigenetic and genetic data may enhance information, albeit nominally, even with small sample sizes. Relieving the burden of multiple testing, developing kernels further to analyze data from multiple omics jointly is well warranted.
\end{abstract}

\section{Background}

The human genome is a highly intricate system comprising various genic and gene regulatory elements. Epigenetic intervention turns it into a jungle. High-throughput technologies have been used to profile phenotypes in multiple omics dimensions. In order to dissect complex genomic traits, statistical tools need to handle a multitude of markers both within and across such dimensions. The kernel score test (KST) enables us to test a set of markers for an overall association with a phenotype [1], such as

\footnotetext{
* Correspondence: summaira.yasmeen@med.uni-goettingen.de ${ }^{1}$ Department of Genetic Epidemiology, University Medical Center, Georg-August University Göttingen, Humboldtallee 32, 37073 Göttingen, Germany

Full list of author information is available at the end of the article
}

those markers within a region of interest (ROI). It highly reduces the burden of multiple testing without simply aggregating the data. KST can be applied to common and rare variants, or adjusted for covariates and applied to data of genome-wide association studies (GWAS), epigenome-wide association studies, or sequence data (where it was named the sequence kernel association test $[2])$.

Our previous research $[3,4]$ focused on genetic data and mainly on logistic regression. In the current analysis, we focused on the use of the KST, employing methylation markers to investigate a normally distributed drug response in independent participants. In several regressions we modeled post treatment log-transformed triglyceride (post-lnTG) as a function of epigenetic and/or genetic 
markers or kernels thereof, adjusting for pre-treatment log-transformed triglyceride (pre-lnTG) and age. We initially investigated the use of KST for epigenetic markers alone and then with genetic markers. We analyzed both the real and simulated data for a selection of candidate ROIs.

\section{Methods}

\section{Data}

The GAW20 data were provided by the Genetics of Lipid Lowering Drugs and Diet Network (GOLDN) study [5], a longitudinal family-based study involving 991 participants of European descent to localize novel loci contributing to triglyceride (TG) response in connection with fat loading and fenofibrate treatment. Data were collected at 4 time points: visits $1,2,3$, and 4 . We performed a pre-/post-treatment analysis using only visits 2 and 4, as the time between visits 1, 2 and 3, 4 was only one 1 day and the pre- and post-methylation data were only recorded at visits 2 and 4 . To normalize distributions, TGs were log-transformed (pre-lnTG and post-lnTG). In addition, we used post-methylation data, GWAS data, and age.

GAW20 also provided simulated data using a family structure and genotypes identical to those in the original data. The answer sheet we were provided described the simulation model. Post-treatment methylation levels were modeled based on pre-methylation with a higher variation at ten $10 \mathrm{CpG}$ cytosine-phosphate-guanine (CpG) markers than for all others. TGs were influenced by five 5 causal SNPs with decreasing heritability and several polygenes. However, the influence of each of the 5 causal SNPs on log TG change decreased with increasing degree of methylation in that particular ROI. Five of the 10 CpG markers were those close to the causal SNPs. We defined the corresponding region as causal ROI and the other mentioned regions as non-causal ROIs.

Since we were focusing only on independent individuals with available phenotypic and genetic/epigenetic information, we only included exactly one member from each pedigree. Hence, we included 150 individuals from the real data and 111 individuals from the simulated data of replicate 84

\section{Selection of ROIs}

We first investigated models with and without kernels with post-treatment methylation data. To define ROIs for the simulated data, we consulted the GAW20 answers for the 5 causal and 5 noncausal CpG markers and associated SNPs to define 10 ROIs with the boundaries lying 0 kilobase pairs (kbp), $15 \mathrm{kbp}$, and $3 \mathrm{kbp}$ upstream and downstream of each of these $5+5 \mathrm{CpG}$ markers. In the real data, we formulated sets of CpG markers in the CPT1A gene defined by intronic boundaries.
All the genomic information is in build hg18. After this we began to investigate genetic markers and used the knowledge of the model for effect simulation.

\section{Regression models and KST}

To investigate the association of specific ROIs with post-lnTGs, we employed linear and semi-parametric kernel regressions, all adjusted for age and pre-lnTG.

In the KST, we used a linear kernel to transform the available epigenetic (or genetic) information of the $n$ individuals into a similarity matrix $K$. This is calculated as $K=\boldsymbol{Z} \boldsymbol{Z}^{t}$, where $\boldsymbol{Z}$ is a $n \times m_{u}$ matrix for $\mathrm{n}$ individuals and $m_{u}$ markers of region $u$. It models a linear effect of the considered markers on the response Y. Let $Y=\left(Y_{1}, \ldots, Y_{n}\right)$ denote the post-lnTGs. $Y$ is modeled as:

$$
\mathrm{Y}=\mathrm{Xb}^{\mathrm{T}}+\mathrm{h}(\mathrm{Z})+\varepsilon
$$

where $X$ is the matrix for known fixed covariates, including age and pre-lnTG; $b$ is the vector of corresponding regression parameters; and $\varepsilon$ denotes the usual residuals. The non-parametric function $h(Z)$ depends on the $n \times n$ dimensional kernel matrix $K$ (for more details refer to Schaid [1]). The KST investigates whether the epigenetic (or genetic) covariance component $h(Z)$ equals zero or not. It is computed from maximum likelihood estimates for the parameters of the null model. The $p$ values were calculated using Davies' exact method [6] with the $\mathrm{R}$ package CompQuadForm [7].

To investigate CpG markers only, we employed a linear regression that included the marker itself, as well as a kernel regression including a kernel for the ROI. For this kernel, we used three different windows, all of which included the $\mathrm{CpG}$ marker itself and windows of

Table 1 Simulated data: association of 10 candidate $\mathrm{CpG}$ markers and their ROls with post-InTG adjusted for pre-InTG and age

\begin{tabular}{|c|c|c|c|c|c|}
\hline \multirow[t]{2}{*}{$\mathrm{ROI}$} & \multirow[t]{2}{*}{ CpG ID } & \multicolumn{3}{|c|}{ KST, Window Size } & \multirow[t]{2}{*}{ Regression } \\
\hline & & $\pm 15 \mathrm{kbp}$ & $\pm 3 \mathrm{kbp}$ & $\pm 0 \mathrm{kbp}$ & \\
\hline $\mathrm{ROI}-1$ & cg00000363 & 0.86 & 0.15 & 0.37 & 0.49 \\
\hline $\mathrm{ROI}-2$ & cg10480950 & 0.09 & 0.09 & 0.58 & 0.63 \\
\hline $\mathrm{ROI}-3$ & cg18772399 & 0.65 & 0.65 & 0.56 & 0.57 \\
\hline ROl-4 & cg00045910 & 0.61 & 0.71 & 0.73 & 0.89 \\
\hline $\mathrm{ROI}-5$ & cg01242676 & 0.49 & 0.33 & 0.49 & 0.57 \\
\hline ROI-6 & cg00703276 & 0.13 & 0.13 & 0.53 & 0.62 \\
\hline $\mathrm{ROI}-7$ & cg01971676 & 0.51 & 0.51 & 0.97 & 0.98 \\
\hline ROI-8 & cg11736230 & 0.79 & 0.83 & 0.22 & 0.18 \\
\hline ROI-9 & cg12598270 & 0.15 & 0.15 & 0.69 & 0.81 \\
\hline ROI-10 & cg00001261 & 0.78 & 0.79 & 0.58 & 0.61 \\
\hline
\end{tabular}


Table 2 Simulation data: association of 5 causal SNPs and their nearest CpG marker with post-InTG, adjusted for pre-InTG and age

\begin{tabular}{llllll}
\hline ROI & CpG ID & SNP ID & CpG Marker & SNP & CpG × SNP \\
\hline ROI-1 & cg00000363 & rs9661059 & 0.0846 & 0.0187 & 0.0484 \\
ROI-2 & cg10480950 & rs736004 & 0.0192 & 0.0237 & 0.0192 \\
ROI-3 & cg18772399 & rs1012116 & 0.1447 & 0.0367 & 0.1933 \\
ROI-4 & cg00045910 & rs10828412 & 0.9252 & 0.4915 & 0.9708 \\
ROI-5 & cg01242676 & rs4399565 & 0.0649 & 0.3519 & 0.0756 \\
\hline
\end{tabular}

$p$ Values of interaction model Eq. 2

sizes $\pm 0 \mathrm{kbp}, \pm 15 \mathrm{kbp}$, and $\pm 3 \mathrm{kbp}$. After investigating CpG markers only, we applied a linear regression including the causal SNP marker $x_{1}$ with its corresponding/ nearest CpG marker $\boldsymbol{x}_{2}$ and their interaction for the 5 causal SNP markers:

$$
\mathbf{Y}=\beta_{0}+\beta_{1} x_{1}+\beta_{2} x_{2}+\beta_{12}\left(x_{1} \times x_{2}\right)+\text { covariates }+\varepsilon
$$

$p$ Values are not adjusted for multiple testing. The significance level is set to $5 \%$. All analyses were performed in R.

\section{Results}

\section{Simulated data}

We performed region-based KST and linear regression to analyze the association of the post-treatment methylation in the 10 candidate ROIs with post-lnTG adjusted for pre-lnTG and age. Table 1 lists the $p$ values for the kernel with the candidate $\mathrm{CpG}$ marker and windows of sizes $\pm 15 \mathrm{kbp}, \pm 3 \mathrm{kbp}$, and $\pm 0 \mathrm{kbp}$, and for single-marker regression analysis. Other than ROI-1, the results for the KST with several CpG markers $( \pm 15 \mathrm{kbp}, \pm 3 \mathrm{kbp})$ are comparable. Furthermore, the results for a single CpG marker (KST $\pm 0 \mathrm{kbp}$, simple regression) are also similar. As to be expected from the simulation, no significant associations were found.

Subsequently, we performed the KST that incorporated genetic information by employing the causal SNPs nearest to the $\mathrm{CpG}$ markers considered above. As expected, this did not yield significant associations (data not shown). Lastly, for the 5 causal ROIs, we employed the regression model defined by eq. (2) with causal SNP, nearest CpG marker, and their interaction. Table 2 presents the results. We found nominally significant associations for the first three SNPs in ROI-1, ROI-2, and ROI-3. The SNPs rs9661059, rs736004, and rs1012116 had the greatest heritabilities of the 5 causal SNPs (see
GAW20 answers). These effects could only be detected by including the nearest CpG marker. Three significant main effects (for rs9661059, rs736004, and rs1012116) and two significant interaction effects were found (Table 2). Our results are in good coherence with the simulation setup of GAW20, in which the effect of the causal SNP is at a maximum in an unmethylated region and decreases as the degree of methylation in the region increases.

\section{Real data}

We analyzed the introns of the CPT1A gene. Table 3 presents all the results. Intron 1 includes the CpG markers previously reported as associated with (baseline) TG [5]. Thus, we investigated single CpG markers of that intron and found two hints for associations with post-lnTG for cg17058475 and cg00574958 (Table 4). Again, the KST and single marker linear regression are in good agreement.

\section{Discussion}

In this analysis, the small sample size that results from using only independent individuals limits our power. Nevertheless, we were still able to detect nominally significant associations for 3 of the 5 causal SNPs from the simulation employing a model of interaction with their nearest $\mathrm{CpG}$ marker. We also found hints for association of cg17058475 and cg00574958 in intron 1 of the CPT1A gene with TGs in the real data by employing KST and the linear regression model. The GOLDN study also reports $\operatorname{cg} 17058475$ and $\operatorname{cg} 00574958$ are also reported by GOLDN study to be as associated with TGs and cg00574958 as correlated with CPT1A expression [5]. Working on the simulated data, we investigated CpG markers in 10 ROIs. A region several kbp in size contains far fewer CpG markers than SNP markers. We revealed for the KST that the window size $0 \mathrm{kbp}$ is similar to linear regression and higher window sizes are similar to each other, yet different from $0 \mathrm{kbp}$. As no direct CpG effect was modeled, no additional conclusions can be drawn. However, the application of the kernel proves feasible with CpG markers, and not only with genetic markers $[3,4]$. Here the use of the kernel is not crucial, as the effect was only given by a simulation model for the causal SNP and the nearest CpG marker, not involving other markers in the region.

The most common design for a treatment-response study is a cohort design with independent people that requires individuals to take the treatment. This might often be unethical for families as a whole (albeit reasonable in

Table 3 Real data: association of sets of CpG markers in 14 introns of the CTP1A gene with post-InTG, adjusted for pre-InTG and age

\begin{tabular}{|c|c|c|c|c|c|c|c|c|c|c|c|}
\hline Intron number(Int) & Int1 & Int3 & int 4 & Int5 & Int 6 & Int7 & Int 9 & $\ln 10$ & $\ln 12$ & $\ln 13$ & Int14 \\
\hline$p$ Value & 0.08 & 0.74 & 0.03 & 0.76 & 0.95 & 0.46 & 0.12 & 0.09 & 0.01 & 0.46 & 0.59 \\
\hline
\end{tabular}

$p$ Values computed by KST 
Table 4 Real data: association of 4 CpG markers in intron 1 of the CPT1A gene with post-InTG, adjusted for pre-InTG and age

\begin{tabular}{lll}
\hline CpG ID & KST & Regression \\
\hline cg00574958 & 0.066 & 0.070 \\
cg09737197 & 0.271 & 0.276 \\
cg17058475 & 0.047 & 0.048 \\
cg01082498 & 0.285 & 0.290
\end{tabular}

$p$ Values computed by KST and single-marker regression

the GOLDN study). As we wanted to see the behavior of kernels and regression in this general context, we opted to focus on unrelated individuals. Our strategy can be adapted for family data. In GAW19, we used KST on family data by introducing a design matrix $-Z c^{T}$ in Eq. (1) - where random effects $c$ caused by familial polygenic background are adjusted for $\mathbf{Y}$ and $Y=$ $X b^{T}+Z c^{T} h(Z)+\varepsilon$ (for more details, see Malzahn et al. [3]). Several GAW20 contributions to this volume used the theoretical or the estimated kinship matrix to construct the random effect in a linear mixed model.

\section{Conclusions}

Our analysis with multi-omics data in a linear regression interaction model in comparison to single omics data in KST and linear regression framework emphasizes that careful integration of multi-omics data might enable researchers to explain a greater proportion of the variance in complex traits, even in small samples. Consequently, it would seem highly warranted to extend kernels to incorporate multiple types of omics data.

\section{Acknowledgements}

The authors thank Andrew Entwistle for editing the manuscript.

\section{Funding}

Publication of this article was supported by NIH R01 GM031575. This research was supported by the German Research Foundation grants DFG: RTG 1644 (publication charges); CRG214: BI 576/2; and PsyCourse (SCHU 1603/7-1; FA241/16-1).

\section{Availability of data and materials}

The data that support the findings of this study are available from the Genetic Analysis Workshop (GAW), but restrictions apply to the availability of these data, which were used under license for the current study. Qualified researchers may request these data directly from GAW.

\section{About this supplement}

This article has been published as part of BMC Proceedings Volume 12 Supplement 9, 2018: Genetic Analysis Workshop 20: envisioning the future of statistical genetics by exploring methods for epigenetic and pharmacogenomic data. The full contents of the supplement are available online at https:// bmcproc.biomedcentral.com/articles/supplements/volume-12-supplement-9.

\section{Authors' contributions}

SY and HB conceived the study and wrote the manuscript. SY, PB, SF, and SP participated in data retrieval and analysis. All authors read and approved the final version of this manuscript.

Ethics approval and consent to participate Not applicable.
Consent for publication

Not applicable.

\section{Competing interests}

The authors declare that they have no competing interests.

\section{Publisher's Note}

Springer Nature remains neutral with regard to jurisdictional claims in published maps and institutional affiliations.

\section{Author details}

'Department of Genetic Epidemiology, University Medical Center, Georg-August University Göttingen, Humboldtallee 32, 37073 Göttingen, Germany. ${ }^{2}$ Department of Psychiatry and Psychotherapy, Ludwig Maximilian University Munich, Nussbaumstrasse 7, 80336 Munich, Germany. Institute of Psychiatric Phenomics and Genomics (IPPG), Medical Center of the University of Munich, Nussbaumstrasse 7, 80336 Munich, Germany.

Published: 17 September 2018

\section{References}

1. Schaid DJ. Genomic similarity and kernel methods I: advancements by building on mathematical and statistical foundations. Hum Hered. 2010;70: 109-31.

2. Wu MC, Lee S, Cai T, Li Y, Boehnke M, Lin X. Rare-variant association testing for sequencing data with the sequence kernel association test. Am J Hum Genet. 2011;89(1):82-93.

3. Malzahn D, Friedrichs S, Bickeböller H. Comparing strategies for combined testing of rare and common variants in whole sequence and genome-wide genotype data. BMC Proc. 2016;10:269-73.

4. Freytag S, Manitz J, Schlather M, Kneib T, Amos Cl, Risch A, Chang-Claude J, Heinrich J, Bickeböller H. A network-based kernel machine test for the identification of risk pathways in genome-wide association studies. Hum Hered. 2016;76(2):64-75.

5. Irvin MR, Zhi D, Joehanes R, Mendelson M, Aslibekyan S, Claas SA, Thibeault KS, Patel N, Day K, Jones LW, et al. Epigenome-wide association study of fasting blood lipids in the genetics of lipid-lowering drugs and diet network study. Circulation. 2014;130(7):565-72.

6. Davies R. Algorithm as 155: the distribution of a linear combination of chi-2 random variables. J R Stat Soc Ser C Appl Stat. 1980;29:323-33.

7. Duchesne P, Lafaye De Micheaux P. Computing the distribution of quadratic forms: further comparisons between the Liu-tang-Zhang approximation and exact methods. Comput Stat Data Anal. 2010;54:858-62.
Ready to submit your research? Choose BMC and benefit from:
- fast, convenient online submission
- thorough peer review by experienced researchers in your field
- rapid publication on acceptance
- support for research data, including large and complex data types
- gold Open Access which fosters wider collaboration and increased citations
- maximum visibility for your research: over $100 \mathrm{M}$ website views per year
At $\mathrm{BMC}$, research is always in progress.
Learn more biomedcentral.com/submissions 